\title{
Simulação no ensino da administração de medicamentos de alta vigilância para enfermeiros: breve visão geral
}

\author{
Simulation in teaching high-alert medication administration for nurses: a brief overview
}

Simulación en la enseñanza de la administración de medicamentos de alto riesgo para enfermeros: una breve descripción general

\section{Resumo}

Objetivou-se apresentar uma breve visão geral sobre o uso da simulação no ensino da administração segura de medicamentos de alta vigilância (MAVs) durante a formação do enfermeiro. Estudos têm reportado baixo conhecimento sobre o uso seguro de MAVs em estudantes de enfermagem e enfermeiros. Como forma de fundamentar esse conhecimento, a realização da simulação durante a formação acadêmica tem o potencial de propiciar o desenvolvimento de segurança bem como a articulação teórico-prática relacionada à atuação em cenários clínicos reais encontrados nos diversos contextos da prática da enfermagem. É notória a consolidação de princípios e conceitos da segurança do paciente, especialmente envolvendo o ensino da administração de MAVs durante a formação do enfermeiro. A simulação pode ser compreendida como uma metodologia ativa de ensino inovadora e pode contribuir na redução de erros de medicação e, consequentemente, promover uma assistência ao paciente mais segura.

Palavras-chave: Simulação; Erros de medicação; Segurança do paciente; Educação superior; Educação em enfermagem.

\footnotetext{
Abstract

The objective was to design a brief overview of the use of realistic simulation in teaching the safe administration of high risk medicine (HRMs) during nursing education. Studies have reported low knowledge about the safe use of HRMs in nursing students and nurses. In support of this knowledge, the realization of realistic simulation during
} 
academic training has the potential to provide the development of safety as well as the theoretical-practical articulation related to acting in real clinical scenarios found in different contexts of nursing practice. The consolidation of patient safety principles and concepts is notorious, especially involving the teaching of HRMs administration during nursing education. Realistic simulation can be understood as an innovative active teaching methodology and can contribute to reducing medication errors and, consequently, promoting safer patient care. Keywords: Simulation technique; Medication errors; Patient safety; Education, higher; Education, nursing.

\section{Resumen}

El objetivo fue diseñar una breve descripción general del uso de la simulación realista en la enseñanza de la administración segura de medicamentos de alto riesgo (MARs) durante la educación de enfermería. Los estudios han informado de un bajo conocimiento sobre el uso seguro de MARs en estudiantes de enfermería y enfermeros. Como forma de sustentar este conocimiento, la realización de la simulación realista durante la formación académica tiene el potencial de brindar el desarrollo de la seguridad así como la articulación teórico-práctica relacionada con la actuación en escenarios clínicos reales encontrados en los diferentes contextos de la práctica de enfermería. La consolidación de los principios y conceptos de seguridad del paciente es notoria, especialmente en lo que respecta a la enseñanza de la administración de MARs durante la formación en enfermería. La simulación realista puede entenderse como una metodología de enseñanza activa innovadora y puede contribuir a reducir los errores de medicación y, en consecuencia, a promover una atención más segura al paciente.

Palabras clave: Simulación; Errores de medicación; Seguridad del paciente; Educación superior; Educación en enfermería.

\section{Introdução}

Erros de medicação (EM) ainda representam um dos incidentes mais comuns e severos nas instituições de saúde e podem ocorrer nas diferentes etapas do sistema de medicação, desde a prescrição até a administração do medicamento ( Keers et al., 2013; Alteren et al., 2018; Santos, Rocha, \& Sampaio, 2019). Mundialmente, a Organização Mundial de Saúde (OMS) lançou no ano de 2017 o terceiro desafio global de segurança do paciente com tema "Medicação sem danos" com o objetivo de reduzir em 50\% os danos graves e evitáveis causados por medicamentos até o ano de 2022 (World Health Organization, 2017). Como prioridade nas ações preventivas, as classes de medicamentos compostas por antimicrobianos (A), potássio (P), insulina (I), narcóticos $(\mathrm{N})$, Chemotherapeutic agents (antineoplásicos - C) e heparinas/anticoagulantes $(\mathrm{H})$ - formando o acrônimo APINCH - foram prioritárias neste desafio por serem considerados medicamentos de alta vigilância (MAVs), os quais são comumente envolvidos em EM e capazes de provocar danos e lesões severas (Institute for Safe Medication Practices (ISMP) (US), 2014; World Health Organization, 2017).

Enfermeiros, na maioria dos serviços de saúde, são os responsáveis pelas etapas finais que permeia o sistema de medicação, ou seja, preparo, administração e monitoramento pós- administração de medicamentos e, com isso, desempenham papel fundamental na prevenção de EM (Keers et al., 2013). Neste sentido, torna-se necessária a construção de estratégias efetivas que possibilitem melhorias na administração segura de medicamentos - em especial de MAVs - sendo o processo de formação do enfermeiro o momento ideal para fundamentar estes conhecimentos.

Todavia, a formação de enfermeiros envolvendo a segurança do paciente, incluindo a administração segura de MAVs, ainda é realizado de modo fragmentado exigindo fortalecimento na articulação teórico-prática durante a graduação (Santos et al., 2020); estudantes requerem uma maior necessidade de conhecimento e treinamento acerca da comunicação de erros, gerenciamento de risco e responsabilização. Desta forma, é necessário que a formação profissional do enfermeiro seja cada vez mais direcionada para competências e habilidades técnicas específicas na área de segurança do paciente, de maneira a propiciar mudanças nas atitudes (comportamentos) dos estudantes, avaliar o processo de trabalho e desenvolver o gerenciamento de erros durante a formação acadêmica (Menezes et al., 2020).

A simulação destaca-se como ferramenta educacional capaz de estimular o desenvolvimento de competências de análise, síntese e tomada de decisão que ultrapassam àquelas relacionadas aos processos clínicos, técnicos e tecnológicos da prática profissional. Além disso, esse método de ensino transpõe as barreiras do treinamento técnico e é capaz de transformar o 
cotidiano por meio de uma prática reflexiva com o aumento da autoconfiança e a redução dos níveis de medo e ansiedade de estudantes em diferentes faixas etárias (Mesquita, Meneses \& Ramos, 2016; Nascimento \& Magro, 2018). Deste modo, compreende-se a importância de se desenvolver conhecimento e competências específicas na área da segurança do paciente, incluindo a administração segura de MAVs em ambiente simulado. Assim, este estudo tem como objetivo apresentar uma breve visão geral sobre o uso da simulação no ensino da administração segura de medicamentos de alta vigilância durante a formação do enfermeiro.

\section{Metodologia}

Estudo elaborado mediante reflexões relacionadas às contribuições da simulação na formação do enfermeiro sobre o ensino da administração segura de MAVs. A proposta do tema surgiu durante a oferta de uma disciplina de um Programa de Pós-Graduação em Enfermagem de uma universidade pública localizada no Centro-Oeste de Minas Gerais desenvolvida entre janeiro e abril de 2021.

\section{Resultados e Discussão}

No atual contexto do cuidado que contemplam atitudes de segurança, o ensino sobre MAVs nas grades curriculares deve ser parte fundamental do processo de formação acadêmica em enfermagem, principalmente, no âmbito da segurança do paciente (Lo et al., 2013). O aprimoramento da segurança por meio de processos educacionais e implantação de sistemas de vigilância é essencial para um cuidado seguro e livre de danos evitáveis (Zanetti et al., 2016).

Erros de medicação podem ser divididos em três principais causas: erros humanos, falhas no sistema e falhas ambientais. Os erros humanos podem envolver falta de competência técnica, pouca aderência aos protocolos de prescrição, pouco conhecimento sobre medicamentos, interpretações incorretas, cansaço, comunicação não efetiva, letras ilegíveis, por exemplo. As falhas no sistema estão relacionadas à notificação insuficiente de eventos adversos envolvendo EM, escassez de bancos de dados para pesquisa sobre a utilização de medicamentos, falta de trabalho em equipe e protocolos de medicamentos ambíguos ou insuficientes. E por fim, as falhas na estrutura ambiental pode favorecer a erros, como: distrações e interrupções durante o processo de preparo e administração de medicamento, pouca iluminação, sobrecarga de trabalho, estresse, ambientes barulhentos, dentre outros (Edwards \& Axe, 2015; Edwards \& Axe, 2018).

Estudo observacional conduzido com enfermeiros identificou conhecimento insuficiente sobre administração de MAVs, relacionado principalmente ao uso de eletrólitos, quimioterápicos e insulina (Santos et al., 2020). Considerando que tais medicamentos apresentam risco elevado de evento adverso devido a erros de administração, faz-se necessário discutir estratégias que fomentam aprendizado reforçado em educações permanentes e também no processo de formação acadêmica do enfermeiro a fim de fortalecer a segurança do paciente (Santos et al., 2020). É importante que a avaliação dos erros aconteça por meio da cultura de aprendizagem e não pela cultura punitiva, que ainda impera nas instituições de saúde. O aprendizado pautado no erro encoraja oportunidades para o compartilhamento de lições aprendidas que fortalecem o processo de ensino contínuo e instiga a possibilidade de abertura da comunicação. Assim, fortalece a cultura de segurança do paciente, além de promover um melhor gerenciamento dos riscos e erros (Castel et al., 2015).

A ausência da temática nos currículos de cursos de graduação da área da saúde, incluindo a enfermagem, dificulta a compreensão dos futuros profissionais em relação à cultura de segurança. Dessa forma, estimula a perpetuação da cultura punitiva no gerenciamento das falhas no processo de cuidados em saúde (Menezes et al., 2020). Alguns princípios básicos de segurança do paciente são recomendados para o desenvolvimento de estratégias que minimizem os erros no processo de medicação, como reduzir a possibilidade de sua ocorrência, torná-los visíveis e reduzir suas consequências. Esses princípios devem ser ancorados em fundamentos na simplificação e padronização de procedimentos (Institute for Safe Medication 
Practices (ISMP), 2014).

Para minimizar falhas, a Agência Nacional de Vigilância Sanitária (ANVISA) publicou um protocolo que contempla os nove certos na administração de medicamentos, como uma das estratégias de prevenção de EM. São eles: paciente certo (utilizar dois identificadores para cada paciente), medicamento certo (confirmar o medicamento com a prescrição e conferir três vezes o rótulo), dose certa, via de administração correta, hora certa, compatibilidade medicamentosa, orientação ao paciente certa, direito a recusar o medicamento e anotação certa (Agência Nacional de Vigilância Sanitária, 2013). Reforçando estas recomendações, estudo internacional discute dez certos para garantir administração de medicamentos de forma mais segura; além dos itens já abordados anteriormente acrescenta-se o item resposta ou resultado certo, ou seja, se o profissional sabe identificar qual resultado esperado a determinado medicamento, possíveis efeitos adversos, reações alérgicas, interações medicamentosas, para que seja possível prestar assistência correta e segura àquele paciente (Edwards \& Axe, 2015).

No processo de formação acadêmica do enfermeiro com intuito de prevenir EM, enfatizando o preparo e a administração de medicamentos de maneira segura, propõe-se como estratégia de ensino uma metodologia ativa baseada na simulação, com abordagem específica do acrônimo APINCH com acréscimo da letra "s" de "Sistemas" no acrônimo com foco direcionado para estratégias de segurança medicamentosa, como é o caso da dupla-checagem para memorização dos principais MAVs. Além disso, é essencial que os docentes instiguem os futuros profissionais na resolução de problemas e desafios encontrados na prática clínica. Para isso, fazem-se necessárias discussões mais robustas sobre a implementação de ferramentas de gerenciamento de riscos, como: educação permanente e continuada da equipe, informações passivas, uso de lembretes ou alertas automáticos nas prescrições médicas, proteção contra falhas, maximização do acesso à informação, acesso limitado ou uso de restrições, padronização e simplificação de processos podem fomentar as reflexões sobre o cuidado de enfermagem acerca das medidas preventivas que envolvem checagem, diluição, preparo e administração de MAVs durante a assistência ao paciente (Institute for Safe Medication Practices (ISMP), 2014).

Neste sentido, a simulação promove por meio da reprodução de cenários e situações fidedignas a contextualização da vivência profissional. Ambientes de simulação garantem a reprodução de aspectos da realidade de forma interativa e dinâmica, com reflexão sobre a solução de problemas do cuidado por meio de embasamento científico, refletindo em impactos positivos na prática assistencial. Entende-se que a participação ativa do estudante nas atividades de simulação possibilita o pensamento crítico e a reflexão sobre as diversas situações práticas. Gera-se, pela vivência prévia da prática, maior segurança para a atuação nos cenários clínicos reais, pois permite, aos estudantes de Enfermagem, uma aproximação com os possíveis casos que encontrarão nos diversos contextos da prática de Enfermagem (Costa et al., 2017).

Segundo a International Nursing Association for Clinical Simulation and Learning (INACSL) ao utilizar a simulação como ferramenta de ensino é necessário atender padrões de boas práticas para alcançar objetivos e resultados compartilhados referente ao tema proposto (INACSL Standards Committee, 2016). Dessa maneira, o método consiste em: briefing, cenários baseados em fatos, planejados e organizados para a sua aplicação, com design detalhado para ser o mais realístico possível e por último, o debriefing. Na construção dos cenários também devem constar: planejamento, objetivo, estrutura, formato, descrição do caso, percepção do realismo, materiais, além de investimento na qualificação dos profissionais que irão atuar como facilitadores de forma que consigam realizar um debriefing problematizador (Cogo et al., 2019; INACSL Standards Committee, 2016; Kaneko \& Baena, 2019). Portanto, quando utilizado essa técnica no ensino sobre MAVs devem ser construídos cenários que abordem o preparo e a administração dos medicamentos com diversidade nas situações do cotidiano vivenciadas nos serviços, os quais devem ser problematizados (Cogo et al., 2019).

Estudo realizado com grupos de acadêmicos de enfermagem de períodos distintos mostrou mais efetividade no raciocínio clínico dos estudantes com maior tempo de formação, supostamente, devido terem mais nível de conhecimento, mais senso de responsabilidade, compromisso ético, além de possivelmente terem mais vivência em cenários de atuação da 
enfermagem através de estágios. Assim, revela a importância de considerar a adequação do conteúdo a cada nível de formação para viabilizar um melhor aproveitamento do método da simulação (Alves et al., 2019). Neste sentido, este método está em ascensão global nos cursos de Graduação em Enfermagem com o objetivo de favorecer melhor formação profissional, diante da importância de praticar, aprender, avaliar ou entender sistemas ou ações humanas. Além disso, a simulação pode propiciar maior interesse, segurança e desenvolvimento dos futuros profissionais possibilitando uma prática mais crítica, criativa e segura (Magnago et al., 2020; Oliveira et al., 2014).

\section{Considerações Finais}

Erros de medicação são capazes de acarretar impactos negativos durante a assistência ao paciente, principalmente envolvendo MAVs. Com isto, demonstra-se a importância da abordagem da temática durante a formação sobre o ensino da administração segura de medicamentos, considerando que falhas nesse processo poderão acarretar incidentes graves. $\mathrm{O}$ uso de cenários realísticos são importantes estratégias de ensino que fomentam ações de prevenção de erros, pois são considerados métodos de formação ativa capazes de contribuir para formação do enfermeiro, aliando de forma crítica, reflexiva e interativa conhecimentos teóricos à prática clínica segura. Visto na literatura e na prática a escassez acerca da temática, é necessário ampliar essa discussão com novos estudos que abordem as contribuições do uso dos cenários de simulação como ferramenta facilitadora de aprendizagem na prevenção de erros evitáveis.

\section{Referências}

Agência Nacional de Vigilância Sanitária. (2013). Assistência segura: uma reflexão teórica aplicada à prática. 41-55. http://www20.anvisa.gov.br/segurancadopaciente/images/documentos/livros/Livro1-Assistencia_Segura.pdf

Alteren, J., Hermstad, M., White, J., \& Jordan, S. (2018). Conflicting priorities: Observation of medicine administration. Journal of Clinical Nursing, 27(1920), 3613-3621. https://doi.org/10.1111/jocn.14518

Alves, N. P., Gomes, T. G., Lopes, M. M. C. O., Gubert, F. do A., Lima, M. A. de, Beserra, E. P., Martins, M. C., \& Cavalcante, V. M. V. (2019). Realistic simulation and its attributes for nurse training. Rev. Enferm. UFPE on Line, 13(5), 1420-1428. https://doi.org/10.5205/1981-8963-v13i05a239014p1420-14282019

Castel, E. S., Ginsburg, L. R., Zaheer, S., \& Tamim, H. (2015). Understanding nurses' and physicians' fear of repercussions for reporting errors: clinician characteristics, organization demographics, or leadership factors? BMC Health Services Research, 15(1), 326. https://doi.org/10.1186/s12913-015-0987-9

Cogo, A. L. P., Lopes, E. de F. S., Perdomini, F. R. I., Flores, G. E., \& Santos, M. R. R. (2019). Building and developing realistic simulation scenarios on safe drug administration. Rev Gaucha Enferm, 40(spe), e20180175-e20180175. https://doi.org/10.1590/1983-1447.2019.20180175

Costa, R. R. O., Medeiros, S. M. de, Martins, J. C. A., Cossi, M. S., \& Araújo, M. S. de. (2017). Percepção de estudantes da graduação em enfermagem sobre a simulação realística. Revista CUIDARTE, 8(3), 1799. https://doi.org/10.15649/cuidarte.v8i3.425

Edwards, S., \& Axe, S. (2015). The 10 "R"s of safe multidisciplinary drug administration. Nurse Prescribing, 13(8), 398-406. https://doi.org/10.12968/npre.2015.13.8.398

Edwards, S. L., \& Axe, S. (2018). Medication management: Reducing drug errors, striving for safer practice. Nurse Prescribing, 16(8), 380-389. https://doi.org/10.12968/npre.2018.16.8.380

INACSL Standards Committee (2016, D. (2016). INACSL Standards of Best Practice: Simulation SM Simulation-Enhanced Interprofessional Education (SimIPE). Clinical Simulation in Nursing, 12, S34-S38. https://doi.org/10.1016/j.ecns.2016.09.011

Institute for Safe Medication Practices (ISMP) (US). (2014). ISMP List of High-Alert Medications in Acute Care Settings. In Institute for Safe Medication Practices (p. 1). https://www.ismp.org/recommendations/high-alert-medications-acute-list

Kaneko, R. M. U., \& Baena, M. H. M. L. (2019). Realistic health care simulation scenario: What is relevant for its design? Revista Da Escola de Enfermagem, 53, e03453. https://doi.org/10.1590/S1980-220X2018015703453

Keers, R. N., Williams, S. D., Cooke, J., \& Ashcroft, D. M. (2013). Causes of medication administration errors in hospitals: A systematic review of quantitative and qualitative evidence. Drug Safety, 36(11), 1045-1067. https://doi.org/10.1007/s40264-013-0090-2

Lo, T. F., Yu, S., Chen, I. J., Wang, K. W. K., \& Tang, F. I. (2013). Faculties' and nurses' perspectives regarding knowledge of high-alert medications. Nurse Education Today, 33(3), 214-221. https://doi.org/10.1016/j.nedt.2012.01.004

Magnago, T. S. B. S., Silva, J. S., Lanes, T. C., Dal Ongaro, J., Luz, E. M. F., Tuchtenhagen, P., \& Andolhe, R. (2020). Simulação realística no ensino de segurança do paciente: relato de experiência. Revista de Enfermagem Da UFSM, 10, e13. https://doi.org/10.5902/2179769236616 
Research, Society and Development, v. 10, n. 12, e523101217576, 2021

(CC BY 4.0) | ISSN 2525-3409 | DOI: http://dx.doi.org/10.33448/rsd-v10i12.17576

Menezes, A. C., Penha, C. S., Amaral, F. M. A., Pimenta, A. M., Ribeiro, H. C. T. C., Pagano, A. S., \& Mata, L. R. F. (2020). Latino Students Patient Safety Questionnaire: cross-cultural adaptation for Brazilian nursing and medical students. Revista Brasileira de Enfermagem, 73(suppl 6). https://doi.org/10.1590/0034-7167-2019-0621

Mesquita, S. K. C., Meneses, R. M. V., \& Ramos, D. K. R. (2016). Metodologias ativas de ensino/aprendizagem: dificuldades de docentes de um curso de enfermagem. Trabalho, Educação E Saúde, 14(2), 473-486. https://doi.org/10.1590/1981-7746-sip00114

Nascimento, M. S., \& Magro, M. C. S. (2018). Realistic simulation: method of improving knowledge and self-confidence of nursing students in the administration of medication. Reme Revista Mineira de Enfermagem, 22, e-1094. https://doi.org/10.5935/1415-2762.20180024

Oliveira, S. N., Prado, M. L., \& Kempfer, S. S. (2014). Use of simulations in nursing education: an integrative review. REME Rev. Min. Enferm, 18(2), 487495. http://www.reme.org.br/artigo/detalhes/941

Santos, G. O., Farre, A. G. M. C., Santana, I. T. S., Rocha, H. M. N., Carvalho, A. A., Santos, G. K. B. B., \& Matos, A. L. P. (2020). Knowledge about the use of potentially dangerous drugs among hospital health care nurses. Rev Rene, 21, e44466. https://doi.org/10.15253/2175-6783.20202144466

Santos, P. R. A., Rocha, F. L. R., \& Sampaio, C. S. J. C. (2019). Ações para segurança na prescrição, uso e administração de medicamentos em unidades de pronto atendimento. Revista Gaucha de Enfermagem, 40(spe), e20180347. https://doi.org/10.1590/1983-1447.2019.20180347

World Health Organization. (2017). Global Patient Safety Challenge: Medication Without Harm. In WHO Global Patient Safety Challenge (p. 16). http://apps.who.int/iris/bitstream/10665/255263/1/WHO-HIS-SDS-2017.6-eng.pdf?ua=1\&ua=1

Zanetti, A. C. B., Gabriel, C. S., Bernardes, A., \& Pereira, L. R. L. (2016). Tradução para português do Brasil e adaptação cultural de um questionário sobre medicamentos potencialmente perigosos. Revista Gaúcha de Enfermagem, 37(3), e59200. https://doi.org/10.1590/1983-1447.2016.03.59200 